\title{
IoT Based Monitoring and Control System using Sensors
}

\author{
${ }^{1}$ Madhura S , ${ }^{2}$ Deepthi G, ${ }^{3}$ Chihnitha B, ${ }^{4}$ Disha D, \\ 1,2,3,4 RV Institute of Technology and Management, Bangalore, India \\ 1'madhu4tuip@gmail.com \\ ${ }^{2}$ deepthig_ec19.rvitm@rvei.edu.in \\ ${ }^{3}$ chihnithab_ec19.rvitm@rvei.edu.in \\ 4dishad is19.rvitm@rvei.edu.in
}

\begin{abstract}
Various physical parameters like humidity, temperature, raindrop, GSM, atmospheric pressure and LDR can be monitored effectively and can be made more interactive with the help of different sensors that are interfaced with microcontrollers like ATmega328P. All the sensors can be connected to this microcontroller ATmega328P as the center preparing unit for the whole framework and plans can be associated with the microcontroller. The realtime monitoring of the various systems becomes possible with this IoT based system. The Paper displays different application based on IoT and proves that the monitoring and control of the system becomes flexible, robust and effective for any real-time implementation .
\end{abstract}

Keywords- Intelligent Transport Systems, Vehicular Ad-hoc Network, Temperature Sensor, Humidity sensor, Gas

\section{INTRODUCTION}

The Internet of things (IoT) is a key technology which illustrates the network of objective things which are implanted by smart sensors, operating system and additional heterogeneous devices whose aim is to connect and exchange information with different devices and software through the Internet. IOT helps in reshaping the current industry into elegant industry prominent with data determined decision creation and promoting a new generation filled with innovative and valuable services [7]. It has an important part in connecting the cyberspace of computing systems to industrial environment which consequently forms a Cyber-Physical System (CPS). It provides a platform to interact with the environment and other objects like human beings over the Internet and also collective intelligence will also improve the process of decision making and empower the people [8]. In the present generation cities are evolving 
smarter by developing Intelligent systems for traffic control, energy management, water management, public transport, automatic street lighting etc. Alongside of providing these services comes a major issue of these systems being attacked upon by some malicious parties when there is an absence of a proper protocol to provide adequate security needed. Smart city usually comprises of collective intelligence usually possessed of Information and Telecommunication Technologies to improve, innovate, and advance a few viable Cloud based IoT implementations which helps to examine, receive, and supervise data in present period and apply them to improve enterprises, cities and localities also improve the process of decision making and empower the citizens to improve quality of life. Some examples of implementation of IOT's are:

- There is a quick response to existent traffic issues decreasing road jamming where traffic lights are linked to get the information from sensors and vehicles thereby regulating the inflection of light.

- The parking space and electric vehicle (EV) imposing docks can communicate with the connected cars and show the drivers the nearest spot available.

- The waste management companies can receive data automatically from Smart garbage cans and can schedule a pick-up when necessary. [9]

- People's mobile phone has become their mobile drivers accredit and identification card with online certification, which is a faster and simple way to access the residential government services is easy as the general public phone is their itinerant driver's license and identification card.

\section{DRONES}

Drones are used for monitoring and measuring air pollution as they are easily navigable. UAVs can monitor traffic, light, noise, wind, temperature, air quality, humidity and many other metrics. They can take off alone in the sky and are in contact with varied operations in inhabitant tasks such as agriculture, transportation, telecommunication, crisis response and ecological preservation. It is all about the payload, so what type equipment is required in the payload is determined by the event which has occurred. Thereby, the most popular drones which are helpful for community security are Da Jiang Innovations (DJI) Inspire and DJI Phantom. [10]. Due to quick development of total residents, the interest for automobile has expanded massively, resolves issues of traffic jamming and avoids accidents. Everyone's living 
is in huge risk, if some mishap happens there's a extended retort time that augments the quantity of passing's, hence a programmed mishap discovery framework must exist to defeat this circumstance. Insights show that driving reason for death by injury is street mishaps. There can be numerous reasons for street mishaps, some of them are, driver carelessness because of tiredness, driving while inebriated, over speeding and so on. A few examinations show that climate conditions can likewise contribute towards the seriousness of a mishap, for example, mist, downpour, high breezes. High breezes can straight forwardly impact the automobile that may stray the vehicle from lane, otherwise in a roundabout way because of danger present on the streets, for example, trees, dividers and so on [5]. Street accidents can be viewed as an impact between any on road vehicles, deterrents, or walkers. The endurance pace of casualty is exceptionally dependent on what amount of time an emergency vehicle requires to arrive at the site of the mishap and afterward convey the patient to the medical clinic. As a rule of street mishaps, the wounds are not extreme and the life of the casualty can be saved, nonetheless because of late appearance of the rescue groups, the wounds turn dangerous. In this manner, the principle objective are to recognize at what position mishap happened, propel the data to the relief groups in significantly a smaller amount of instance, so that essential moves, to spare the living of victim is taken care of [6].

Intelligent Transport Systems (ITS) in view of Internet of Things (IoT) which are receiving mainstream and be able to be viewed as an answer for development in the street security. One successful procedure to diminish traffic disasters and rescue valuable life's and diminishes the reaction period after a mishap has occurred. Critical examination is been done to address this problem and to reduce the reaction time following a mishap. Various methodologies are in support of this cause. In a unique situation, VANET (Vehicular Ad-hoc Network) is used in which each running auto mobile goes about the same as a hub. On event of mishap, the alarm notifications are conveyed by means of Radio Frequency section. Single methodology utilizes boundary changes to identify a mishap, GSM (Global System for Mobile Communication) which is utilized to deliver an alarm notification and region of mishap is followed by GPS (Global Positioning System) component. Cell phone-based framework which utilizes an android application to recognize accidents are likewise projected. This framework determines the modification of slope point by methods for an accelerometer sensor, the pace by methods for GPS and gives a caution on location of mishap [18-21]. A few frameworks center on 
preventive technique in light of the fact that toward the end, objective is to spare lives. This framework especially Centre's around the security of bikes and checks if the driver is lazy. One procedure Centre's around utilizing the accelerometer sensor keeps observing the automobile pace and notifies a mishap as it comes to beneath the edge end. A new methodology points on forestalling tipsy driving by introducing liquor sensors on the directing haggle permitting the rider to ride if he is inebriated by means of estimating the liquor percentage in his oxygen [6].

\section{WEATHER MONITORING SYSTEM USING SMART SENSORS BASED ON IOT}

For example, GSM SIM900A, voltage controller, and pneumatic stress sensor (BMP180 sensor) which are given below.The microcontroller applies six climate boundaries using each and every sensor in an IoT dominant climate observation frame. The following sensors are used to transmit data via the internet through IoT.

- Temperature and dampness sensor (DHT 11 sensor), light sensor,

- Downpour surface sensor,

- Contamination sensor (MQ-135 gas sensor),

- Pneumatic force sensor (BMP180)

To send data within the boundaries via the web we utilize the GSM SIM900A module and it is more than once rehashed throughout a period stretch. At that point clients need to take a gander at a portion of the internet destinations to perceive information [12]. The dampness and temperature information from the DHT11 sensor is measured by microcontroller [MCU]. This is then transmitted to The GSM SIM 900A module, a Wi-Fi module, that is one of the significant Internet stages. It can transmit information to IoT cloud, with the decrease in the expense of gadgets supporting $\mathrm{Wi}-\mathrm{Fi}$, this pattern quickens. With cloud administrations, individuals can utilize this information to impart in an assortment of patterns, for example, utilize chime or send them an email or sending a SMS. As referenced before, IoT just permits human-to-human connection however doesn't collaborate with the human-to-gadget and gadget to-gadget communication. Nearby levels and significant changes in energy utilization will turn into an essential piece of any nation's energy strategy [14]. Internet of things (IoT) is a connected amount climate which is associated with the Internet. In IoT, objects are vehicles using a center screen or implicit sensor. For e.g., objects are given to IP locations and they 
possess the ability to accumulate information on the organization. Innovation installed in systems helps cooperate along with the center, state or outside climate, which influences decisions. Settled in, (for example, sensors) by gadgets, Internet availability and different sorts of equipment can interrelate with others on the web and IoT can control questionably. The table shows the specialized changes in the data trade in affinities of the Internet (IoT) [1]

\section{i. Implementation}

IoT-based climate observing framework with brilliant sensors finishes usage arrangement, and parts can be portrayed in Table 1. The total execution arrangement of IoT-based climate checking framework with keen sensor [13].

In IoT-based climate observing framework using brilliant sensors, it utilizes extraordinary parameters, for example, GSM SIM900A, pneumatic stress sensor (BMP180 sensor) and voltage controller, which are given below in table 1 :

\begin{tabular}{|c|c|c|}
\hline & Table1: & Rating of components \\
\hline S.NO & Components & Description \\
\hline 1 & GSMSIM900A & $\begin{array}{l}\text { Operating voltage }+5 \mathrm{~V} \text {, } \\
\text { Use for establishing communication }\end{array}$ \\
\hline 2 & microcontroller & $\begin{array}{l}\text { supply voltage }+5 \mathrm{~V} \text {, CPU type } 8 \text {-bit AVR, pin } \\
\text { count } 28 \text { max count. Operating frequency } 20 \mathrm{MHz}\end{array}$ \\
\hline 3 & Voltage regulator & Power supply $+5 \mathrm{~V}$, use a simple feed-forward \\
\hline 4 & DHT11 sensor & $\begin{array}{l}\text { Operating voltage } 3.5-5 \mathrm{~V} \text {, used for temperature } \\
\text { and humidity }\end{array}$ \\
\hline 5 & Raindrops sensor & Operating voltage $5 \mathrm{~V}$, used for detection of rain \\
\hline 6 & LDR sensor & Operating voltage $5 \mathrm{~V}$, used for light \\
\hline 7 & MQ-135 gas sensor & Operating voltage $5 \mathrm{~V}$, used for air pollution \\
\hline 8 & BMP180 sensor & Operating voltage $5 \mathrm{~V}$, used for air pressure \\
\hline
\end{tabular}

\section{ii. GSM SIM 900A}

A GPS or GSM module is a chip or circuit that utilizes to discover correspondence amongst a cell phone and processing machine. The global norm for portable that is utilized for correspondence over a extensive reach. In a GSM/GPRS modem, you have an interior TCP/IP stack to permit the web to interface with the GPRS. It is fitting for SMS, information, voice base exchange demands in the M2M interface's SIM900A module [2, 3, 4]. 


\section{TEMPERATURE AND HUMIDITY SENSOR}

The computerized signal yield is highlighted by the DHT11 temperature and humidity sensor. It is designed with an 8-cycle microcontroller that is superior. The high quality and spectacular long-haul sound are ensured by its invention. It has remarkable consistency, rapid response, hostile to the potential of obstruction and elite. Each DHT11 sensor contains an extremely detailed dampness change chamber alignment. The orientation coefficients stored in the memory of the OTP software, inbuilt sensors simultaneously differentiate signals, we can name those coefficients of adjustment. In order to turn out to be snappy and simple, the single-wire sequential interface system is orchestrated. the little scale, low force, isolation of signal propagation up to 20 meters, empowering a variety of uses and even the most challenging. The product is a 4-pin bundle of single line pins. Exceptional packages may be offered by consumers wanting a supportive association $[2,3,4]$.

\section{RAINDROP SENSOR}

The unit of the raindrop sensor is a basic device for detecting downpour. It can be used quite well as a switch when raindrop falls through the pouring board and also for precipitation power estimation. In the estimation of precipitation, the basic yield is used in finding drops. Gracefully associated with $5 \mathrm{~V}$ power, the LED light to turn on when the acceptance board has no downpour fall, and the performance of DO is high. The performance of DO is poor when dropping a small amount of water, the switch pointer with turn on. The water pellets are dismissed, and when returned to the underlaying condition, the level is elevated. An exchange system implemented by precipitation is a downpour switch or raindrop sensor [2, 3, 4].

\section{VOLTAGE REGULATOR AND POLLUTION SENSOR}

The device that supplies electric power catalyst that transfers electromagnetic forces electronically basically a power supply machine. The basic ability of a control source is to transform a type of electrical energy into various structures, so it is often referred to as an electrical energy into various structures. So, it is often referred to as an electrical converter 
[11]. Customer hardware gracefully uses small and independent force AC cables or different types of electrical blocks divider tablets, or chargers. For electrical devices needing electricity, the $\mathrm{AC}$ connector is used but the force does not provide internal fixings to achieve voltage and force. Interior force is for example, used gracefully for inward or inside, flexibly internal circuit configuration. A $12 \mathrm{VDC}$ connector for running the arrangement in this paper $[2,3,4]$.

A sensor of air pollution is a system that enables and channels air contamination in the quick territory. It can be used for the internal and external environment at present. At present, these sensors can be carried out at home or purchased from some finished merchandise. Sensors have generally been extremely selective, but with specialized turn of events, the population is more moderate and fills in these sensors more. Such sensors will help fill various needs beyond the natural eye circle and help concentrate on natural problems. The contents of MQ135 and SnO2, and this is an exceptional patch. For example, Mq-135 gas sensor include alkali nitrogen oxygen, liquor, vertical mix, sulphur, and smoke $[2,3,4]$.

\section{BMP180 Sensor}

For gas or fluid weight measurement, the pressure is often a glance at the force required to forestall a fluid, and the BMP180 gauge incorporate weight, temperature, and size for the most part, as shown by the metric per unit field. From [2] The use of the framework requires the microcontroller ATmega328P as the core planning unit for the entire framework, and the microcontroller can be compatible with all sensors and plans. The following pin is DHT11, an information pin, which may apply to the stickiness and temperature statures of the microcontrollers up to stick 5. Pins 1 and 4 DHT11 are Vcc and Gnd, and there is no relation between Pin 3.

The gaseous stress sensor sticks that are connected with the 28 and 27 pin microcontrollers are the sda and scl pins. The fourth pin and air pollution sensor are Vcc and Gnd, the third pin has no relation, the information pin is the second pin of the air contamination sensor, and the 26pin microcontroller will give the contamination message. Vcc and Gnd are pins 1 and 4 of the downpour stage modules, the third pin has no connection, and the second pin of the downpour module is a knowledge and understanding pin that can give downpour calculations to the microcontrollers of 24 and 23 pins or not. The second (Rx) and third (Tx) devices are associated with the GSM SIM900A's Tx and Rx sticks. 


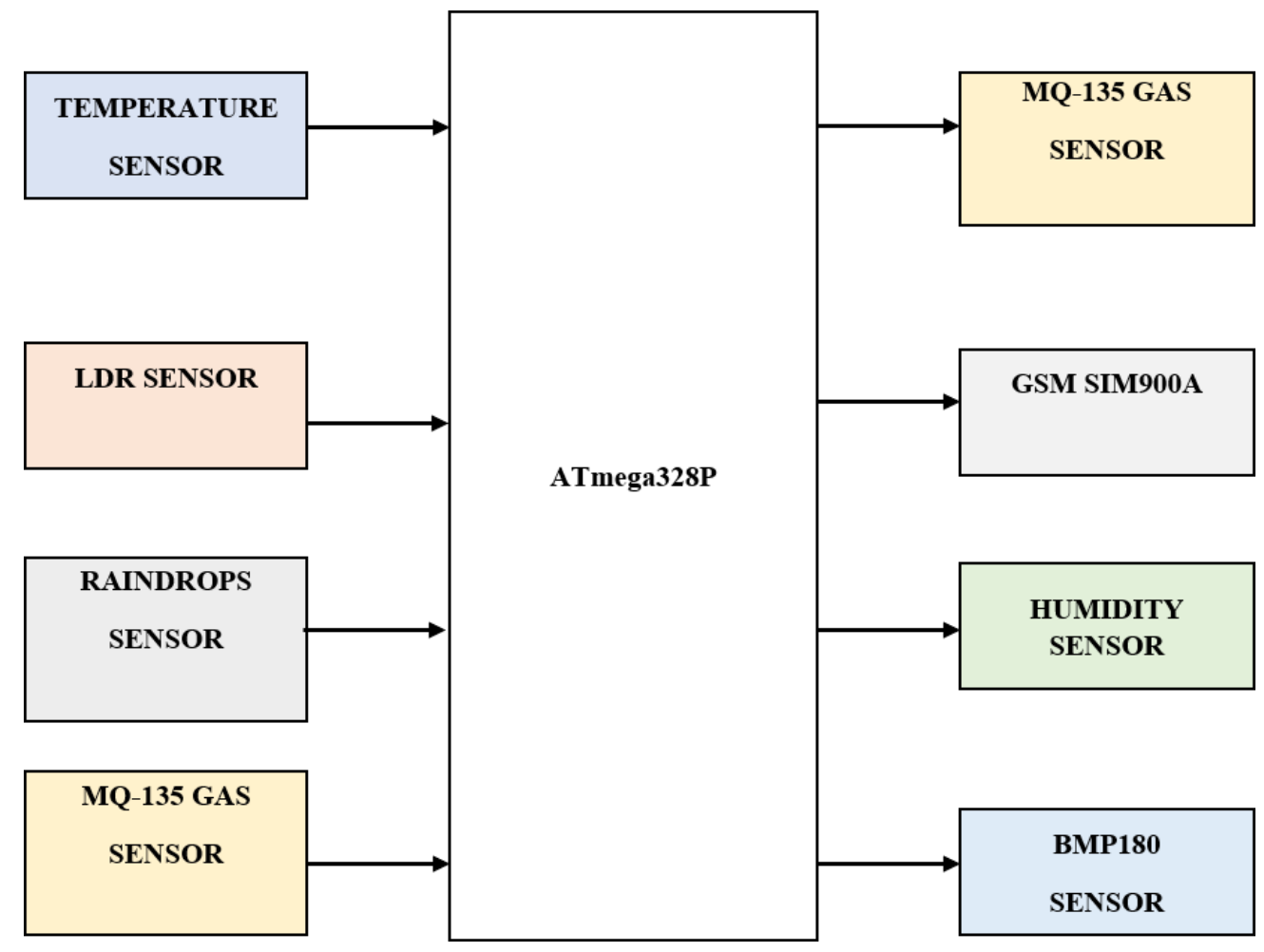

Fig 1: Implementation block of sensors with ATmega328P

\section{CONCLUSION}

The sensors mentioed above can be used for collection and analysis of data like temperature, humidiy, atmospheric pressure, LDR, GSM, Gas and raindrop. These data can be implemented for real-time data analysis with the help of IoT and microcontroller as detailed in the paper. These latest sensors are more efficient and reliable for use on the modern sensing hardware for better control of electronic devices. These sensors not only serve as data collectors but also can be implemented to alert the system for good disaster management. The future work involves the development of the proposed work on the hardware platefrom and analyase the results.

\section{REFERENCES}

[1] Yashaswi Rahut, Rimsha Afreen, Divya Kamini , " Smart weather monitoring and real time alert system using IoT”, International Research Journal of Engineering and Technology (IRJET), Volume: 05 Issue: 10 , Oct 2018 
[2] Bulipe Srinivas Rao, K. Srinivasa Rao, N. Ome, “ Internet of Things (IOT) Based Weather Monitoring system", International Journal of Advanced Research in Computer and Communication Engineering ISO 3297:2007 Certified Vol. 5, Issue 9, September 2016

[3] Ferdin Joe John Joseph, “ IoT Based Weather Monitoring System for Effective Analytics”, International Journal of Engineering and Advanced Technology (IJEAT) ISSN: 2249 8958, Volume-8 Issue-4, April, 2019

[4] Elie Nasr, Elie Kfoury, David Khoury, “An IoT Approach to Vehicle Accident Detection, Reporting, and Navigation”. [online]

[5] Sreenithy Chandran, Sneha Chandrasekar, Edna Elizabeth N, “ Konnect: An Internet of Things(IoT) based Smart Helmet for Accident Detection and Notification”, IEEE Annual India Conference (INDICON), 2016, DOI: 10.1109/INDICON.2016.7839052

[6] Mohammed Abdul Kader, Md. Eftekhar Alam, Sabrina Momtaj, Saidun Necha, Mohammed Shamsul Alam, Abdul Kadar Muhammad Masum," IoT Based Vehicle Monitoring with Accident Detection and Rescue System”, 22nd International Conference on Computer and Information Technology (ICCIT), 18-20 December, 2019

[7] Iot-based smart cities: A surveyhttps://ieeexplore.ieee.org/abstract/document/7555867

[8] Adaptive and context-aware service composition for IoT-based smart citieshttps //www.sciencedirect.com/science/article/abs/pii/S0167739X16308688

[9] Challenges and Opportunities of Waste Management in IoT-Enabled Smart Cities https://ieeexplore.ieee.org/abstract/document/7892970

[10] Survey on Collaborative Smart Drones and Internet of Things for Improving Smartness of Smart Cities https://ieeexplore.ieee.org/abstract/document/8795473

[11] Park, E.; Del Pobil, A.P.; Kwon, S.J. The Role of Internet of Things (IoT) in Smart Cities: Technology Roadmap-oriented Approaches. Sustainability 2018, 10, 1388 https://www.mdpi.com/2071-1050/10/5/1388\#cite

[12] 2.Janssen, M., Luthra, S., Mangla, S., Rana, N.P. and Dwivedi, Y.K. (2019), "Challenges for adopting and implementing IoT in smart cities: An integrated MICMACISM approach", Internet Research, Vol. $29 \quad$ No. 6, pp. 15891616. https://doi.org/10.1108/INTR-06-2018-025 
[13] 3.Kamel Boulos, M.N., Al-Shorbaji, N.M. On the Internet of Things, smart cities and the WHO Healthy Cities. Int J Health Geogr 13, 10 (2014). https://doi.org/10.1186/1476072X-13-10

[14] 4.A. Sharif, J. Li, M. Khalil, R. Kumar, M. I. Sharif and A. Sharif, "Internet of things — smart traffic management system for smart cities using big data analytics," 2017 14th International Computer Conference on Wavelet Active Media Technology and Information Processing (ICCWAMTIP), Chengdu, 2017, pp. 281-284, doi: 10.1109/ICCWAMTIP.2017.8301496.

[15] Mugunthan, S. R. "Decision Tree Based Interference Recognition for Fog Enabled IOT Architecture." Journal of trends in Computer Science and Smart technology (TCSST) 2, no. 01 (2020): 15-25.

[16] Chen, Joy Iong Zong, and S. Smys. "Interoperability Improvement in Internet of Things Using Fog Assisted Semantic Frame Work." Journal of trends in Computer Science and Smart technology (TCSST) 2, no. 01 (2020): 56-68.

[17] Bhalaji, N. "Reliable Data Transmission with Heightened Confidentiality and Integrity in IOT Empowered Mobile Networks." Journal of ISMAC 2, no. 02 (2020): 106-117.

[18] Raj, Jennifer S. "Improved Response Time and Energy Management for Mobile Cloud Computing Using Computational Offloading." Journal of ISMAC 2, no. 01 (2020): 38-49.

[19] Smys, S., and G. Ranganathan. "Performance Evaluation of Game Theory Based Efficient Task Scheduling For Edge Computing." Journal of ISMAC 2, no. 01 (2020): 5061.

[20] Smys, S. "A Novel Multi-Tier Architecture Based Mobile Cloud Computing For Enhanced Energy Utilization." Journal of ISMAC 2, no. 01 (2020): 62-72.

[21] Chen, Joy Iong Zong. "Optimal Multipath Conveyance with Improved Survivability for WSN's In Challenging Location." Journal of ISMAC 2, no. 02 (2020): 73-82. 